\title{
ON AN EXAMPLE OF AHERN AND RUDIN
}

\author{
JOHN T. ANDERSON
}

(Communicated by Clifford J. Earle, Jr.)

\begin{abstract}
We show that the polynomial hull of a certain totally real threesphere in $\mathbb{C}^{3}$ constructed by Ahern and Rudin is the union of a two-parameter family of analytic disks.
\end{abstract}

\section{INTRODUCTION}

If $M$ is a compact real $n$-dimensional submanifold of $\mathbb{C}^{n}$ then $M$ must have nontrivial polynomial hull $\widehat{M}=\left\{z \in \mathbb{C}^{n}:|P(z)| \leq \max _{M}|P|\right.$ for all polynomials $P\}$; a result of Alexander [3] states that the topological dimension of $\widehat{M} \backslash M$ is at least $n+1$. One would like to understand $\widehat{M}$, perhaps by finding analytic disks with boundaries in $M$ (an analytic disk $\Delta$ is the image of an analytic map $f:\{z \in \mathbb{C}:|z|<1\} \rightarrow \mathbb{C}^{n}$; if $f$ extends continuously to $\{|z| \leq 1\}$, and if $\partial \Delta=f(|z|=1) \subset M$, then by the maximum principle $\Delta \subset \widehat{M})$. If $n=2$ and $M$ has a complex tangent at $p \in M$, i.e., the tangent space to $M$ at $p$ has a nonzero complex subspace, then generically either the tangent is of hyperbolic type and $M$ is locally polynomially convex, or the tangent is of elliptic type, in which case a technique of Bishiop [6] can be used to construct analytic disks with boundaries in $M$ near $p$. In the same paper, Bishop showed that for any $M \subset \mathbb{C}^{2}$ diffeomorphic to the two-sphere there are at least two points of compex tangency. However, a theorem of Gromov $[9, \mathrm{p}$. 193] guarantees the existence of embedded three-spheres in $\mathbb{C}^{3}$ that are totally real (no complex tangents). In this case, different methods must be used to exhibit analytic structure in $\widehat{M} \backslash M$. Ahern and Rudin [1] gave the first explicit example of such a sphere, as a graph over the boundary of the ball in $\mathbb{C}^{2}$,

$$
\left.M=\left\{z_{1}, z_{2}, \bar{z}_{1} z_{2} \bar{z}_{2}^{2}+i z_{1} \bar{z}_{1}^{2} \bar{z}_{2}\right):\left|z_{1}\right|^{2}+\left|z_{2}\right|^{2}=1\right\} .
$$

They did not address the question of determining $\widehat{M}$. Recently Forstneric [7] constructed a totally real three-sphere in $\mathbb{C}^{3}$ for which he was able to produce a one-parameter family of analytic disks in $\widehat{M} \backslash M$.

The goal of this paper is to show that polynomial hull of the Ahern-Rudin example is foliated by analytic disks.

Received by the editors January 21, 1991; presented at the 865th AMS Meeting, Tampa, FL, March 22, 1991.

1991 Mathematics Subject Classification. Primary 32E20. 
Theorem 1. Let $M$ be the totally real three-sphere given by (1.1). There exists a two-parameter family $\Delta_{\theta_{1}, \theta_{2}}$ of analytic disks such that

$$
\widehat{M} \backslash M=\left\{\bigcup_{\theta_{1}, \theta_{2}} \Delta_{\theta_{1}, \theta_{2}}\right\} \cup \Delta_{1} \cup \Delta_{2}
$$

where $\Delta_{1}=\left\{\left|z_{1}\right|<1, z_{2}=z_{3}=0\right\}$ and $\Delta_{2}=\left\{\left|z_{2}\right|<1, z_{1}=z_{3}=0\right\}$.

The proof uses a result of Wermer that seems to be particularly useful for constructing analytic disks when $M$ is invariant under certain group actions (see also $[5,8]$ ). The arguments used here parallel closely those in [10], where disks in $\mathbb{C}^{2}$ invariant under $(z, w) \rightarrow\left(z e^{i \theta}, w e^{-i \theta}\right)$ are studied.

In related work, Alexander [4] has proved that if $M$ is a graph in $\mathbb{C}^{3}$ of a function $f$ continuous on the boundary of the ball $B$ in $\mathbb{C}^{2}$ then $\widehat{M}$ covers $B_{2}$; i.e., the projection of $\widehat{M}$ to $\mathbb{C}^{2}$ is $\bar{B}_{2}$. He also gives conditions on $f$ under which $\widehat{M}$ is itself a graph over $B_{2}$, but his results do not apply to the case considered here. Finally, Ahern and Rudin [2], by different methods than those employed here, have recently generalized our result by describing the hull of a totally real three-sphere $M$ in $\mathbb{C}^{3}$ of the form $\{(z, w, \Gamma(z \bar{z}) / z w):(z, w) \in$ $b B\}$ where $\Gamma$ belongs to a certain class of plane curves. In particular, they show that $\widehat{M} \backslash M$ is both a graph over $B$ and a union of analytic disks.

\section{Proof of Theorem 1}

We note that $M$ is invariant under the transformation

$$
z=\left(z_{1}, z_{2}, z_{3}\right) \rightarrow T_{\theta_{1}, \theta_{2}}(z)=\left(z_{1} e^{i \theta_{1}}, z_{2} e^{i \theta_{2}}, z_{3} e^{-i\left(\theta_{1}+\theta_{2}\right)}\right) .
$$

Clearly $\widehat{M}$ must also be $T_{\theta_{1}, \theta_{2}}$-invariant. Let $F(z)=z_{1} z_{2} z_{3}$. Then $F$ 。 $T_{\theta_{1}, \theta_{2}}=F$. If $z \in M$ then $F(z)=\left|z_{1}\right|^{2}\left|z_{2}\right|^{2}\left(\left|z_{2}\right|^{2}+i\left|z_{1}\right|^{2}\right)$. Let $\gamma$ be the image of $M$ under $F$. Setting $\left|z_{1}\right|=r$, we obtain a parametrization of $\gamma$ : if

$$
\gamma(r)=r^{2}\left(1-r^{2}\right)\left(1-r^{2}+i r^{2}\right)
$$

then $\gamma=\{\gamma(r): 0 \leq r \leq 1\} . \gamma$ is a simple closed analytic curve in the complex plane, with a double point at the origin and no other singularity. Let $\Omega$ be the region bounded by $\gamma$. If $\zeta \in \gamma \backslash\{0\}$ then it is easy to check that there exists a unique $r \in(0,1)$ so that $\gamma(r)=\zeta$. This $r$ we denote by $r(\zeta)$. If $z \in M$ and $F(z)=\zeta \neq 0$, then the orbit of $z$ under the transformations $T_{\theta_{1}, \theta_{2}}$ is the torus $\left\{T_{\theta_{1}, \theta_{2}}\left(z_{r}\right): 0 \leq \theta_{1}, \theta_{2}<2 \pi\right\}$ where $z_{r}=\left(r, \sqrt{1-r^{2}}, \gamma(r) / r \sqrt{1-r^{2}}\right)$ and $r=r(\zeta)$. If $F(z)=0$ then $z_{1}=0$ or $z_{2}=0$ and the orbit of $z$ is the circle $z_{1}=z_{3}=0,\left|z_{2}\right|=1$ in the first case, or $z_{2}=z_{3}=0,\left|z_{1}\right|=1$ in the second case.

Lemma 1. $F(\widehat{M})=\bar{\Omega}$.

Proof. Clearly $F(\widehat{M}) \subset \bar{\Omega}$. If $\zeta_{0} \in \Omega$ and $\zeta_{0} \notin F(\widehat{M})$, then $h(z)=$ $\left(F(z)-\zeta_{0}\right)^{-1}$ is an element of $P(M)$. If $g=\sum_{\alpha} A_{\alpha} z^{\alpha} \quad\left(\alpha=\left(\alpha_{1}, \alpha_{2}, \alpha_{3}\right)\right)$ is any polynomial in $\left(z_{1}, z_{2}, z_{3}\right)$, then there exists a polynomial $\tilde{g}$ in one variable so that for $0<r<1$,

$$
\tilde{g}(\gamma(r))=\frac{1}{4 \pi^{2}} \int_{0}^{2 \pi} \int_{0}^{2 \pi} g \circ T_{\theta_{1}, \theta_{2}}\left(z_{r}\right) d \theta_{1} d \theta_{2} .
$$


In fact we can take $\tilde{g}(\zeta)=\sum a_{j} \zeta^{j}$ where $a_{j}=A_{(j, j, j)}$. Since $h$ is constant on the orbit of $z_{r}$, for any polynomial $g$ we can write

$$
\tilde{g}(\gamma(r))-\left(\gamma(r)-\zeta_{0}\right)^{-1}=\frac{1}{4 \pi^{2}} \int_{0}^{2 \pi} \int_{0}^{2 \pi}\left((g-h) \circ T_{\theta_{1}, \theta_{2}}\right)\left(z_{r}\right) \theta_{1} d \theta_{2}
$$

and thus

$$
\max _{0 \leq r \leq 1}\left|\tilde{g}(\gamma(r))-\left(\gamma(r)-\zeta_{0}\right)^{-1}\right| \leq \max _{z \in M}|g(z)-h(z)|
$$

(extending the inequality by continuity to $r=0$ ). Thus if $h \in P(M)$, $\left(\gamma(r)-\zeta_{0}\right)^{-1}$ is uniformly approximable by polynomias on $\gamma$. It follows that $\left(\zeta-\zeta_{0}\right)^{-1}$ is uniformly approximable by polynomials in $\zeta$ on $\Omega$, which is false. The proof is complete.

Now we use a theorem of Wermer [10]: let $A$ be a uniform algebra on a compact Hausdorff space $X$, and let $M_{A}$ denote the maximal ideal space of $A$. For $f \in A$, let $\hat{f}$ denote the Gelfand transform of $f$. Define for $g \in A$ and $\zeta \in \hat{f}\left(M_{A}\right)$,

$$
\Psi(\zeta)=\log \left(\max \left\{|g(p)|: p \in M_{A} \text { and } \hat{f}(p)=\zeta\right\}\right) .
$$

Then Wermer's Theorem states that $\Psi$ is subharmonic on $\mathbb{C} \backslash \hat{f}\left(M_{A}\right)$. If for $\zeta \in \Omega$ we set

$$
Z_{i}(\zeta)=\max \left\{\left|z_{i}\right|: z \in \widehat{K} \text { and } F(z)=\zeta\right\}, \quad i=1,2,3,
$$

this result applied to the algebra $A=P(M)$ of uniform limits of polynomials on $M$ implies that $\log Z_{i}$ is subharmonic on $\Omega$. We will construct analytic disks in $\widehat{M}$ using the functions $\log Z_{i}$, which we will show are actually harmonic in $\Omega$. First we must examine the boundary behavior of the $Z_{i}$. We need

Lemma 2. Suppose $z^{0} \in M$ and $F\left(z^{0}\right)=\zeta^{0} \in \gamma \backslash\{0\}$. Then $z^{0} \in M$.

Proof. Assume $z^{0} \notin M$. Let $r \in(0,1)$ such that $F\left(z^{0}\right)=\gamma(r)$. Let $T$ be the orbit of $z_{r}$ under the $T_{\theta_{1}}, \theta_{2} . T$ is easily seen to be polynomially convex. Since $z^{0} \notin T$, there exists a polynomial $P$ with $\left|P\left(z^{0}\right)\right|>1,|P|<1$ on $T$. Choose a neighborhood $U$ of $T$ in $M$ with $|P|<1$ on $U$. The image of $M \backslash U$ under $F$ is a closed subarc $\tilde{\gamma}$ of $\gamma$ exlcuding $\zeta^{0}$. There exists $g$ analytic on $\Omega$, continuous on $\bar{\Omega}$, and $\delta>0$, so that $g\left(\zeta^{0}\right)=1$ and $|g|<1-\delta$ on $\tilde{\gamma}$. For any $n, Q(z)=(g \circ F(z))^{n} P(z)$ is a uniform limit of polynomials on $M \cup\left\{z^{0}\right\}$. On $U,|Q| \leq|P|<1$, while on $M \backslash U,|Q|<(1-\delta)^{n}|P|<1$ for sufficiently large $n$. But $\left|Q\left(z^{0}\right)\right|=\mid P\left(z^{0}\right)>1$, which contradicts $z^{0} \in \widehat{M}$ and completes the proof.

Next we use Lemma 2 to show that $Z_{1}$ continuously assumes the boundary values $r(\zeta)$ on $\gamma \backslash\{0\}$. Let $\left\{\zeta_{n}\right\}$ be a sequence in $\Omega$ with $\zeta_{n} \rightarrow \zeta \in \gamma \backslash\{0\}$. Choose $z^{(n)} \in \widehat{M}$ with $F\left(z^{(n)}\right)=\zeta_{n}$ and $\left|z_{1}^{(n)}\right|=Z_{1}\left(\zeta_{n}\right)$. We can assume $z^{(n)}$ converges to $z \in \widehat{M}$. Then $F(z)=\zeta$. By Lemma 2, $z \in M$. Thus $Z_{1}\left(\zeta_{n}\right) \rightarrow$ $\left|z_{1}\right|=r(\zeta)$. Similarly we can show that $Z_{2}$ and $Z_{3}$ continuously assume the boundary values $\sqrt{1-r^{2}}$ and $\gamma(r) / r \sqrt{1-r^{2}}$, respectively, on $\gamma \backslash\{0\}$. Now we need a regularity result on solutions of the Dirichlet problem on $\Omega$ when the boundary data satisfies certain estimates. The proof of the following lemma is essentially contained in the proof of Lemma 4 of [10] and the discussion preceding it. 
Lemma 3. Let $G$ be subharmonic on $\Omega$, and suppose $g(\zeta)=\lim _{\zeta^{\prime} \rightarrow \zeta} G\left(\zeta^{\prime}\right)$ exists and is a continuous function of $\zeta$ on $\gamma \backslash\{0\}$. Furthermore suppose there exist positive constants $c, C$ so that $C>g(\zeta)>c \log |\zeta|$ for all $\zeta \in \gamma \backslash\{0\}$. For $\zeta_{0} \in \Omega$ set

$$
\widetilde{G}\left(\zeta_{0}\right)=\int g(\zeta) d \mu_{\zeta_{0}}(\zeta)
$$

where $d \mu_{\zeta_{0}}$ is harmonic measure at $\zeta_{0}$ with respect to $\Omega$. Then $\widetilde{G}$ is harmonic and bounded above on $\Omega$, assumes continuously the boundary values $g$ on $\gamma \backslash\{0\}$, and $G(\zeta) \leq \widetilde{G}(\zeta)$, for all $\zeta \in \Omega$.

Now define

$$
\begin{aligned}
U_{1}\left(\zeta_{0}\right) & =\int \log r(\zeta) d \mu_{\zeta_{0}}(\zeta) \\
U_{2}\left(\zeta_{0}\right) & =\int \log \left(\sqrt{1-r^{2}(\zeta)}\right) d \mu_{\zeta_{0}}(\zeta), \\
U_{3}\left(\zeta_{0}\right) & =\int \log |\zeta|-\log r(\zeta)-\log \left(\sqrt{1-r^{2}(\zeta)}\right) d \mu_{\zeta_{0}}(\zeta) \\
& =\log \left|\zeta_{0}\right|-U_{1}\left(\zeta_{0}\right)-U_{2}\left(\zeta_{0}\right) .
\end{aligned}
$$

Apply Lemma 3 with $(G, g)=\left(\log Z_{1}, \log r\right),\left(\log Z_{2}, \log \left(\sqrt{1-r^{2}}\right)\right.$, and $\left(\log Z_{3}, \log |\zeta|-\log r(\zeta)-\log \left(\sqrt{1-r^{2}(\zeta)}\right)\right.$ in turn. In each case the estimate required on $g$ follows from the fact that $|\zeta|=|\gamma(r(\zeta))| \leq r(\zeta)^{2}\left(1-r(\zeta)^{2}\right)$. We obtain

$$
\begin{aligned}
& \log Z_{1}(\zeta) \leq U_{1}(\zeta), \\
& \log Z_{2}(\zeta) \leq U_{2}(\zeta), \\
& \log Z_{3}(\zeta) \leq \log |\zeta|-U_{1}(\zeta)-U_{2}(\zeta),
\end{aligned}
$$

where the functions on the right-hand side are harmonic on $\Omega$.

Lemma 4. For all $\zeta \in \Omega, \log Z_{1}(\zeta)=U_{1}(\zeta), \log Z_{2}(\zeta)=U_{2}(\zeta)$, and $\log Z_{3}(\zeta)$ $=\log |\zeta|-U_{1}(\zeta)-U_{2}(\zeta)$.

Proof. If any one of these three inequalities should fail then, by (2.2),

$$
\log Z_{1}(\zeta)+\log Z_{2}(\zeta)+\log Z_{3}(\zeta)<\log |\zeta| .
$$

But clearly $Z_{1}(\zeta) Z_{2}(\zeta) Z_{3}(\zeta) \geq|\zeta|$, so we have a contradiction.

Now let $V_{i}(\zeta)$ be the harmonic conjugate in $\Omega$ of $U_{i}(\zeta), i=1,2$. Set

$$
\varphi_{i}=e^{U_{i}+\sqrt{-1} V_{i}} \quad i=1,2 .
$$

Then each $\varphi_{i}$ is analytic and nonvanishing on $\Omega$. Set

$$
\phi(\zeta)=\left(\varphi_{1}(\zeta), \varphi_{2}(\zeta), \frac{\zeta}{\varphi_{1}(\zeta) \varphi_{2}(\zeta)}\right)
$$

$\phi$ maps $\Omega$ analytically into $\mathbb{C}^{3}$. We claim $\phi(\Omega) \subset \widehat{M}$. Fix $\zeta \in \Omega$. Note $F(\phi(\zeta))=\zeta$ and, by Lemma $4,\left|\varphi_{1}(\zeta)\right|=e^{U_{1}(\zeta)}=Z_{1}(\zeta)$. Choose $z \in \widehat{M}$ with $F(z)=\zeta$ and $\left|z_{1}\right|=Z_{1}(\zeta)$. Then $\varphi_{1}(\zeta)=e^{i \theta_{1}} z_{1}$ for some $\theta_{1} \in[0,2 \pi)$. If $\log \left|z_{2}\right|<U_{2}(\zeta)$ then

$$
\begin{aligned}
\log |\zeta| & =\log \left|z_{1}\right|+\log \left|z_{2}\right|+\log \left|z_{3}\right| \\
& <U_{1}(\zeta)+U_{2}(\zeta)+\log \left|z_{3}\right|=\log |\zeta|-\log Z_{3}(\zeta)+\log \left|z_{3}\right|
\end{aligned}
$$


by Lemma 4 . But then $\log Z_{3}(\zeta)<\log \left|z_{3}\right|$, a contradiction. Thus $\log \left|z_{2}\right|=$ $U_{2}(\zeta)$, which implies $\left|z_{2}\right|=\left|\varphi_{2}(\zeta)\right|$, and so $z_{2}=\varphi_{2}(\zeta) e^{i \theta_{2}}$ for some $\theta_{2}$. Finally,

$$
z_{3}=\frac{\zeta}{z_{1} z_{2}}=\frac{\zeta e^{-i\left(\theta_{1}+\theta_{2}\right)}}{\varphi_{1}(\zeta) \varphi_{2}(\zeta)}
$$

and so $\phi(\zeta)=T_{\theta_{1}, \theta_{2}}(z) \in \widehat{M}$.

Define for $\left(\theta_{1}, \theta_{2}\right) \in[0,2 \pi) \times[0,2 \pi)$ the analytic disk $\Delta_{\theta_{1}, \theta_{2}}$ to be the image of $\Omega$ under $T_{\theta_{1}, \theta_{2}} \circ \phi$. It is easy to verify that $\Delta_{\theta_{1}, \theta_{2}}$ and $\Delta_{\theta_{1}^{\prime}, \theta_{2}^{\prime}}$ are disjoint unless $\left(\theta_{1}, \theta_{2}\right)=\left(\theta_{1}^{\prime}, \theta_{2}^{\prime}\right)$. We have shown that $\Delta_{0,0} \subset \widehat{M}$, so $\Delta_{\theta_{1}, \theta_{2}}$ is a two-parameter family of disks lying in $\widehat{M}$. Let $\Delta_{1}, \Delta_{2}$ be as in the statement of Theorem 1. Since $\partial \Delta_{1}$ and $\partial \Delta_{2}$ are contained in $M, \Delta_{1} \cup \Delta_{2} \subset \widehat{M}$. To complete the proof of Theorem 1 we must show that each $z \in \widehat{M} \backslash M$ is contained in some $\Delta_{\theta_{1}, \theta_{2}}$ or in $\Delta_{1} \cup \Delta_{2}$. By Lemma 2, either $F(z)=\zeta \in \Omega$, or $F(z)=0$. In the first case, we must have $\left|z_{i}\right|=Z_{i}(\zeta), i=1,2,3$. If not, by Lemma 4, $\left|z_{1} z_{2} z_{3}\right|<\left|Z_{1}(\zeta) Z_{2}(\zeta) Z_{3}(\zeta)\right|=|\zeta|$, a contradiction. Thus $z=T_{\theta_{1}, \theta_{2}} \circ \phi(\zeta) \in \Delta_{\theta_{1}, \theta_{2}}$ for some $\left(\theta_{1}, \theta_{2}\right)$. In the second case, if $z \notin \Delta_{1} \cup \Delta_{2}$, take a neighborhood $U$ in $M$ of the polynomially convex set $\partial \Delta_{1} \cup \partial \Delta_{2}$ and a polynomial $P$ so that $|P(z)|>1$ while $|P|<1$ on $U$. Then argue as in the proof of Lemma 2, taking $\zeta^{0}=0$, to conclude that $z \notin \widehat{M}$, a contradiction. We are finished with the proof of Theorem 1.

\section{ACKNOWLEDGMENT}

I wish to thank W. Rudin and the referee for pointing out several errors in a preliminary version of this paper.

\section{REFERENCES}

1. P. Ahern and W. Rudin, Totally real imbeddings of $S^{3}$ in $\mathbb{C}^{3}$, Proc. Amer. Math. Soc. 94 (1985), 460-462.

2. _ Hulls of 3-spheres in $\mathbb{C}^{3}$, preprint, 1991.

3. H. Alexander, A note on polynomial hulls, Proc. Amer. Math. Soc. 33 (1972), 389-391.

4. __, Polynomial hulls of graphs, Pacific J. Math. 147 (1991), 201-212.

5. J. Anderson, Polynomial hulls of sets invariant under an action of the special unitary group, Canad. J. Math. 40 (1988), 1256-1271.

6. E. Bishop, Differentiable manifolds in complex euclidean space, Duke J. Math. 32 (1965), $1-21$.

7. F. Forstneric, A totally real three-sphere in $\mathbb{C}^{3}$ bounding a family of analytic disks, Proc. Amer. Math. Soc. 108 (1990), 887-892.

8. T. Gamelin, Hartogs series, Hartogs functions, and Jensen measures, Spaces of Analytic Functions, Kristiansand 1975, Lecture Notes in Math., vol. 512, Springer-Verlag, Berlin and New York, 1976.

9. M. L. Gromov, Partial differential relations, Springer-Verlag, Berlin and New York, 1986.

10. J. Wermer, Subharmonicity and hulls, Pacific J. Math. 58 (1975), 283-290.

Department of Mathematics, College of the Holy Cross, Worcester, Massachusetts 01610-2395

E-mail address: janderson@hcacad.holycross.edu 